\title{
Designing, Development and Formulation of Mouth Disintegrating Telmisartan Tablet with Extended Release Profile Using Response Surface Methodology
}

\author{
Muhammad Abdullah Akram ${ }^{1}$, Taha Nazir ${ }^{1,2,3 *}$, Nida Taha ${ }^{3}$, Adeel Adil ${ }^{4}$, Muhammad Sarfraz ${ }^{5}$ and Saeedur Rasheed Nazir ${ }^{1}$ \\ ${ }^{1}$ Faculty of Pharmacy, University of Sargodha, Sargodha 40100 Pakistan \\ ${ }^{2}$ Biochemistry, Chemical Pathology, Molecular Biology Research Group, UMC\&RC, University of Sargodha, Sargodha 40100 Pakistan \\ 3Intellectual Consortium of Drug Discovery \& Technology Development Inc., Saskatoon Saskatchewan S7L3W8 Canada \\ ${ }^{4}$ Department of Biosciences, COMSATS Institute of Information Technology, Islamabad, PO; 45550, Pakistan \\ ${ }^{5}$ Department of Pharmacy, Islamia University of Bahawalpur, Bahawalpur, Pakistan
}

\begin{abstract}
The Mouth Disintegrating Tablets (MDT's) is continuously growing in clinical practice. The pharmaceuticals researchers are exploring the formulations for extended release dosage form with optimal therapeutic benefits. Hence, we aimed this study to develop the mouth disintegrating telmisartan tablet with extended release profile using response surface methodology. Mouth Disintegrating Extended Release Tablets (MDERT's) was prepared with the intention of obtaining immediate as well as sustain therapeutic effect. The MDERT's characterized with different determinants. The results were tabulated to illustrate the drug release curves of all 6 formulations upto 12 hrs, DSC spectra of Telmisartan, Kyron T134 ${ }_{134}$, Primogel, Telmisartan + Kyron T1 $134_{134}$ + Primogel, Chitosan, CMC and different excepients. The response surface models were developed for disintegration time, wetting time, water absorption ratio and cumulative $\%$ drug release $(10 \mathrm{~min})$ to determine the significant variance. The cumulative release \%age of Telmisartan formulation F2 was comparatively important because of having $\geq 80 \%$ cumulative release. Moreover, a least volume of media $17 \mathrm{ml}$ was used by F2 formulation. Whereas, Fourier Transform Infrared Spectroscopy (FT-IR) and DSC studies were executed for any possible chemical interaction or incompatibility in drugs, polymers and excipients used in intended formulations. Additionally, the wetting and dispersion time for F2 were also in targeted rang of 52 and 44 seconds respectively, indicating the rapid disintegration of Telmisartan to produce desired therapeutic effects. Hence, in conclusion, the determinants of MDERTS are adjustable within acceptable range to enhance the efficiency. An extended release profile using response surface methodology may also be designed to formulate the MDT's to render the dose, regimen, protocol and frequency of hypertensive patient in clinical practice.
\end{abstract}

Keywords: Telmisartan; Mouth disintegrating tablet; Extended release profile; Response surface methodology

\section{Introduction}

At present, in research and modern technology, new and innovative techniques are emerging up in the field of Drug Delivery System. Now we shall discuss such new and modern drug delivery system with dual character i.e. or dispersible as well as having extended release profile in order to enhance patient compliance. However, as the demand for Mouth Disintegrating Tablets (MDT’s) continues to grow [1], scientists are exploring ways to adapt MDT formulations for drugs that require extended release profile for optimal therapeutic benefits [2].

In addition of that, there are certain techniques to manufacture the Mouth Disintegrating Tablets, the Center of Drug Evaluation and Research (CDER) Nomenclature Standards Committee (in 1998) defined an orally disintegrating tablet (ODT) as "Solid dosage form containing medicinal substances which disintegrates rapidly, usually within a matter of seconds, when placed upon the tongue" (Food and Drug Administration) [3]. Some of the new advanced technologies which are commonly being used in last few decades are Freeze drying/ Lyophilization, Molding, Direct Compression, Cotton Candy Process, Spray Drying, Sublimation and Mass Extrusion [4].

There are remarkable techniques for the preparation of such dosage forms with dual characteristic profile, fast release as well as sustained release. MDERTs produced revolutionary changes in the field of new drug delivery by sustaining the therapeutic effect of administered dosage form upto $12 \mathrm{hrs}$ that was not easily possible before. There has been a remarkable increase in the interest in extended release dosage form, due to prohibitive cost of developing new drug entities, discovery of the new polymers and improvement in efficiency and safety provided by these. SRDDS is a modified dosage form that prolongs the therapeutic activity of the drug. Accordingly, a pro-drug or analogue modification of the drug sustains blood level is considered as extended release system. Several terms have been used to describe the various types of dosage forms to provide long duration of action.

The Superdisintegrants are agents or mixture of agents that when added to the formulations enhance the crumbling and disintegration of solid dosage forms into very tiny particles as compared to conditions when superdisintegrants are not present. Disintegrants have strong resistance against the binding ability of binders and all physical forces that result in tablet formation under compression. Rate limiting step in the rapid drug release is thought to be tablet disintegration.

*Corresponding author: Taha Nazir, Research Associate, Biochemistry, Chemica Pathology \& Molecular Biology Research Group, University Medical \& Diagnostic Centre, University of Sargodha, Sargodha, Pakistan, 40100, Tel: 03212220885; E-mail: taha.nazir@uos.edu.pk

Received July 12, 2015; Accepted September 02, 2015; Published September 09, 2015

Citation: Akram MA, Nazir T, Sarfraz M, Nazir SR (2015) Designing, Development and Formulation of Mouth Disintegrating Telmisartan Tablet with Extended Release Profile Using Response Surface Methodology. J Bioequiv Availab 7: 262266. doi: $10.4172 / \mathrm{jbb} .1000250$

Copyright: @ 2015 Akram MA, et al. This is an open-access article distributed under the terms of the Creative Commons Attribution License, which permits unrestricted use, distribution, and reproduction in any medium, provided the original author and source are credited. 
Citation: Akram MA, Nazir T, Sarfraz M, Nazir SR (2015) Designing, Development and Formulation of Mouth Disintegrating Telmisartan Tablet with Extended Release Profile Using Response Surface Methodology. J Bioequiv Availab 7: 262-266. doi:10.4172/jbb.1000250

Disintegrants are the agents when formulated in formulation cause them to melt rapidly when comes in contact with aqueous media [5]. Mucilage's have dual property of binding and disintegrant depending upon its concentration within the dosage form. Usually $1-10 \%$ concentration of total weight can serves as disintegrant.

Telmisartan with its brand name MICARDIS angiotensin receptor antagonist and non-peptide drug. Telmisartan is chemically formulated as 4-[(1,4-dimethyl-2-propyl[2,6-bi-1H-benzimidazol]-1yl)methyl 1]-[1,1-biphenyl]2-carboxylic acid with its empirical formula is $\mathrm{C}_{33} \mathrm{H}_{30} \mathrm{~N}_{4} \mathrm{O}_{2}$ and molecular weight is 514.63 and structural formulae is as follows (Figure 1).

Telmisartan in pure form is whitish in color to slightly yellowish solid. It Telmisartan is nor soluble in water. Telmisartan is having $\mathrm{pH}$ range of 3 to 9 and shows solubility in base (strong). The Standard dose of Telmisartan is available in $20 \mathrm{mg}, 40 \mathrm{mg}$ and $80 \mathrm{mg}$ potencies. Telmisartan is angiotensin used to treat high blood pressure and also to control other factors i.e. Congestive heart failure, Strokes problems, and Heart problems leading to death. Such dosage form maybe prescribed by doctors for other concerning problems.

\section{Materials and Methods}

This project of designing, development and formulation of mouth disintegrating Telmisartan tablet with extended release profile using response surface methodology was conducted in High Tech Lab., Faculty of Pharmacy, University of Sargodha, Pakistan. The major materials used in during experimental work were the Telmisartan (Highnoon Laboratories), Carboxy methyl cellulose (Vision pharmaceuticals), Mg-stearate and talc (Willson pharmaceuticals), Lactose (welcare pharmaceutical Sargodha), Chitosan (Highnoon Laboratories), Kyron T134 ${ }_{134}$ (Qantar pharmaceuticals), Primogel (Bosch pharmaceuticals), Polyvinyl Pyrrolidone (S.B pharmaceuticals), Saccharine sodium and flavors (Saffron Laboratories). While, the major equipments used were the FTIR (IR Prestige 21 Shimadzu), Thermal Analyzer (SDT Q600), Oven (IM-30 IRMECO Gmb), Spectrophotometer UV 1700 (Shimadzu), Micropipettes, $\mathrm{pH}$ meter (Jenway 3510), Friabilator (Pharma test, Germany), Weight balance (Shimadzu A x200), Dissolution Apparatus (Pharma test) and Disintegration Apparatus (Pharma test).

\section{Direct compression method}

All the desired materials such as drug, polymers, superdisintegrants and other excipients required for extended release orodispersible tablets were weighed accurately on scientific electronic weighing balance as shown in Tables 1 and 2 .

Extended release and Orodispersible, two portions of all of the six formulations were designed. The materials from portion 1 were

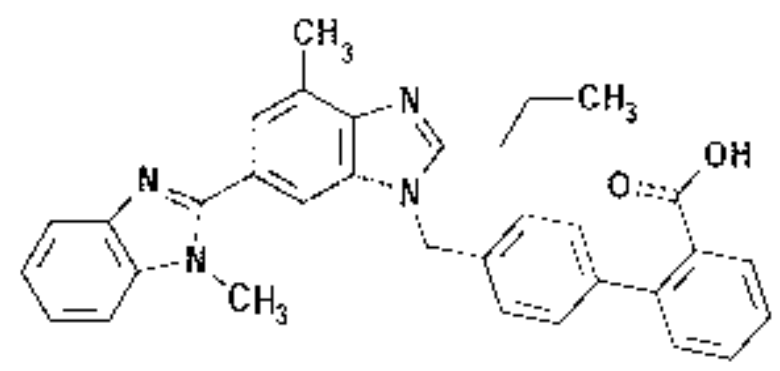

Figure 1: Telmisartan.

\begin{tabular}{|l|l|l|l|l|l|l|}
\hline Ingredients $\mathbf{( m g )}$ & F1 & F2 & F3 & F4 & F5 & F6 \\
\hline Telmisartan & 40 & 40 & 40 & 40 & 40 & 40 \\
\hline CMC & 20 & - & - & 10 & & 10 \\
\hline Chitosan & - & 20 & - & 10 & 20 & \\
\hline PVP & - & - & 20 & - & & 10 \\
\hline Mg-Stearate & 4 & 4 & 4 & 4 & 4 & 4 \\
\hline Talc & 4 & 4 & 4 & 4 & 4 & 4 \\
\hline Lactose & 82 & 82 & 82 & 82 & 82 & 82 \\
\hline Total Weight(mg) & 150 & 150 & 150 & 150 & 150 & 150 \\
\hline
\end{tabular}

Table 1: Extended release portion of Formulation.

\begin{tabular}{|l|c|c|c|c|c|c|}
\hline Ingredients (mg) & F1 & F2 & F3 & F4 & F5 & F6 \\
\hline Telmisartan & 40 & 40 & 40 & 40 & 40 & 40 \\
\hline Pre-gelatinize Starch & 16 & - & 12 & 8 & 4 & \\
\hline Kyron T 134 & - & 16 & 4 & 8 & 12 & \\
\hline Mg-Stearate & 4 & 4 & 4 & 4 & 4 & 4 \\
\hline Talc & 4 & 4 & 4 & 4 & 4 & 4 \\
\hline Lactose & 77 & 77 & 77 & 77 & 77 & 93 \\
\hline Orange Flavor & 5 & 5 & 5 & 5 & 5 & 5 \\
\hline Saccharine Sodium & 4 & 4 & 4 & 4 & 4 & 4 \\
\hline Total Weight(mg) & 150 & 150 & 150 & 150 & 150 & 150 \\
\hline
\end{tabular}

Table 2: Mouth disintegrating portion of Formulation.

weighed properly on electrical balance for 06 formulations. Then they were passed through sieve No 40. After sieving these were homogenize in tumbling cylindrical mixer for 10 minutes at a speed of $15 \mathrm{rpm}$. Then a wet mass of this mixture was prepared by adding a suitable amount of water to produce granules. Granules were dried at the temperature of $60^{\circ} \mathrm{C}$ over night. Then granules were brought so that to be passed through a sieve of mesh No. 80 to receive granules of desired size. After this we prepared $2^{\text {nd }}$ portion, in which all materials were weighed properly on electrical balance for 06 formulations. Then they were passed through sieve no 40 . After sieving these were homogenize in tumbling cylindrical mixer for 10 minutes at a speed of $15 \mathrm{rpm}$.At the end we mixed both portions of weighing materials for 06 formulations to homogenize in tumbling cylindrical mixer for 20 minutes and compressed into extended release orodispersible tablets by using ZP 19 station rotary tablet compressing machine [6].

\section{Pre and post compression determinants}

The flow properties were investigated by measuring the bulk and tapped density. Other precompression determinants were Bulk density, Tapped Density, Angle of Repose AOR, Carr's Compressibility Index, and Hausner's Ratio. While the Post-compression determinants were Thickness, Weight variation, Hardness and Friability, Wetting Time, Water Absorption Ratio, Drug Content, In Vitro Drug Release, and In Vitro Disintegration Time.

\section{Study design}

MDERTS dosage form was prepared with ambition of to get dual effects; producing immediate as well as sustain therapeutic effect. Direct compression method used for it. The major components of this preparation were Telmisartan, Carboxy methyl cellulose polyvinyl Pyrrolidone, Chitosan, Talc, Mg-stearate, Lactose. While the equipments and instruments used to study the parameters were FTIR (IR Prestige 21 Shimadzu), Thermal Analyzer (SDT Q600), Oven (IM-30 IRMECO Gmb), Spectrophotometer UV 1700 (Shimadzu), Micropipettes, pH meter (Jenway 3510), Friabilator (Pharma test, Germany), Weight balance (Shimadzu A x200), Weight balance (Shimadzu A x200), Disintegration Apparatus (Pharma test), 
Citation: Akram MA, Nazir T, Sarfraz M, Nazir SR (2015) Designing, Development and Formulation of Mouth Disintegrating Telmisartan Tablet with Extended Release Profile Using Response Surface Methodology. J Bioequiv Availab 7: 262-266. doi:10.4172/jbb.1000250

Aluminum foil, Platinum loop, Spatula, Beakers. Finally, we have characterized the MDERTS dosage form with different determinantsBulk density, Tapped density, Angle of repose, Carrs index, Hausners ratio, Thickness, Weight variation, Hardness, Weight variation, Wetting time, Wetting volume, Water absorption ratio, Drug content, In vitro drug release, In vitro disintegration time etc. The final results of Angle of Repose, Bulk density, Tapped density, Hausner's Ratio and Carr's Index, Hardness, Weight variation, Thickness, Friability, Disintegration time and Wetting time has been tabulated to illustrate the finding. While, the drug release curves of all 6 formulations upto $12 \mathrm{hrs}$, DSC spectra of Telmisartan, Kyron $\mathrm{T}_{134}$, Primogel, Telmisartan + Kyron $\mathrm{T}_{134}+$ Primogel, Chitosan, CMC and different excepients are presented as comprehensive scientific figures.

\section{Statistical analysis}

The computer software SPSS used for statistical ANOVA (Analysis of Variance) analysis. That is applied to design, develop and formulate be best possible mouth disintegrating telmisartan tablet with extended release profile using response surface methodology.

\section{Results}

The response surface models were developed for disintegration time, wetting time, water absorption ratio and cumulative $\%$ drug release $(10 \mathrm{~min})$ to determine the significant variance. The cumulative release percentage of Telmisartan formulation F2 was comparatively important because of having $\geq 80 \%$ cumulative release. Moreover, a least volume of media i.e. $17 \mathrm{ml}$ was used by F2 formulation. Whereas, Fourier Transform Infrared Spectroscopy (FT-IR) and DSC studies confirmed that there was no any chemical interaction and no incompatibility among drug, polymers and excipients used in intended formulations. Wetting and dispersion time were least $52 \mathrm{sec}$ and $44 \mathrm{sec}$ respectively for F2 indicating rapid disintegration and release of drug.

\section{Fourier transform infrared (FTIR) spectroscopy}

Fourier transform infrared spectroscopy all the excipients and pure were taken alone and in combinations. The FTIR spectra of Telmisartan alone and in combination with superdisintegrants and polymers were taken to check any interaction between drug molecules as well as with superdisintegrants. The spectrum of FTIR had shown that there was complete presence of characteristic peaks of drug molecules and excipients. It means that there was no interaction between and drug excipients (Figures 2-4).

The FTIR spectrum of pure Telmisartan showed the prominent peak of the drug at $3400 \mathrm{~cm}^{-1}$ of $\mathrm{O}-\mathrm{H}$ stretching of $-\mathrm{COOH}$ acid and other peaks at $2960 \mathrm{~cm}^{-1}$ due to $\mathrm{C}-\mathrm{H}$ stretching vibration of aromatic group, $-\mathrm{OH}$ bending and $-\mathrm{C}=\mathrm{O}$ stretching of $-\mathrm{COOH}$ acid at $1385 \mathrm{~cm}$

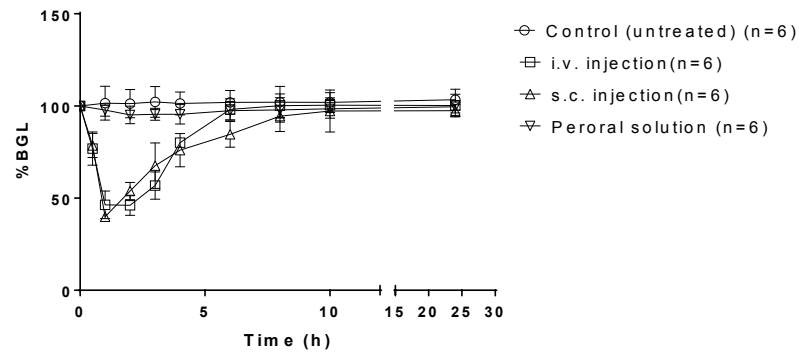

Figure 2: FTIR Spectra of Telmisartan

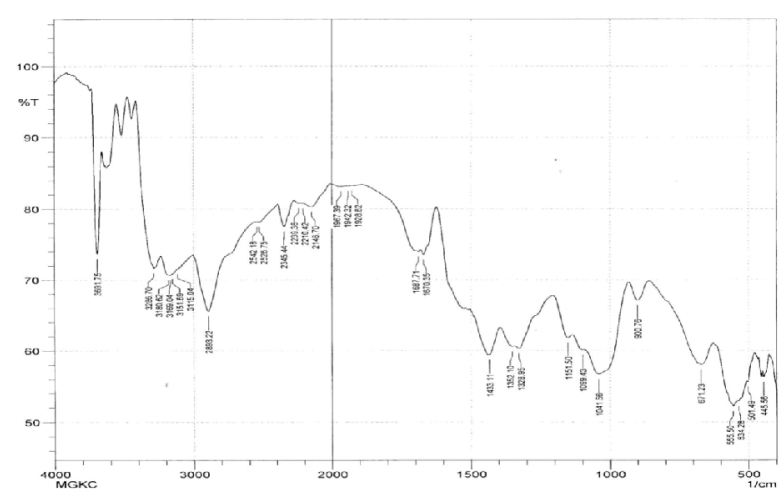

Figure 3: FTIR spectra Telmisartan+Kyron T134134+Primogel.

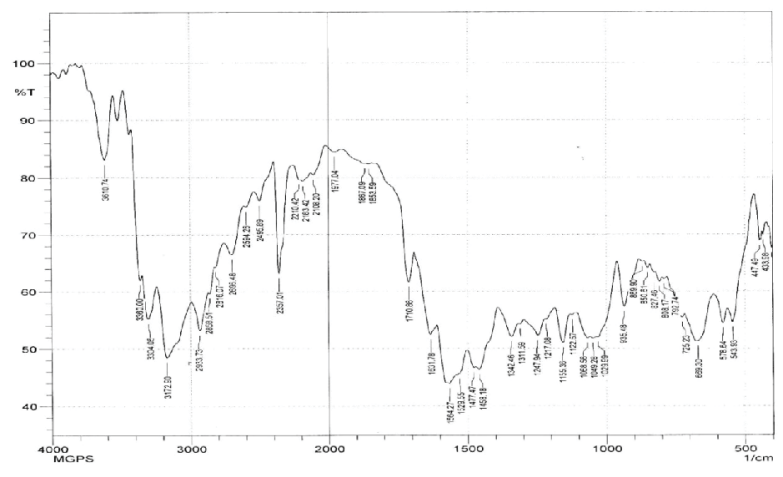

Figure 4: FTIR spectra of Telmisartan + chitosan + PVP + CMC

${ }^{1}, \mathrm{C}=\mathrm{C}$ stretching of aromatic group at $1600 \mathrm{~cm}^{-1}$ and functional group of (-COOH) at $1697 \mathrm{~cm}^{-1}$. FTIR spectra of Telmisartan with excipients and excipients with each other also shown that same characteristic peak were present as in single spectra of drug and excipients.

\section{Differential scanning calorimetry studies}

DSC studies of drugs molecules and excipients were carried out alone as well as in combination. The characteristic peaks of alone drugs and with excipients were compared. This study also confirms that there was no significant interaction among excipients and drug. DSC studies were conducted to check interaction among drug and superdisintegrants and polymers. The typical DSC thermogram data was scanned. Its examination proved that there was no incompatibility among drug and excipients used for different formulations. The whole study was conducted on SDT. Q 600 Thermal Analyzer USA at increasing temperature rate of $10^{\circ} \mathrm{C} / \mathrm{min}$ for specific time period for a particular agent (Figure 5).

\section{Drug release studies}

Cumulative percentage drug release was upto $49.5 \%$ for formulation F2 among all formulations. F2 contains Kyron $\mathrm{T}_{134}$ as super disintegrants which shows that Kyron $\mathrm{T}_{134}$ had better disintegration properties as compared to primogel. This result was also confirmed by formulations F3, F4 and F5.These formulations contain Kyron $\mathrm{T}_{134}$ and primogel in combination in different ratios. Their release was less than formulation F2 that contains only Kyron $\mathrm{T}_{134}$ as superdisintegrants and greater than F1 that contains primogel as super disintegrant. The disintegrant nature of Kyron $\mathrm{T}_{134}$ was also confirmed by making a formulation F6 that has no primogel or Kyron $\mathrm{T}_{134}$ and its drug release was very low as 
compared to other formulations. Release data of all 06 formulations had also shown that chitosan has best retarding properties than PVP and CMC. Formulations contained chitosan alone and in combination with PVP and CMC had produced best orodispersible extended release effect of drug as compared to formulations containing PVP and CMC alone. The order in which superdisintegrants and polymers had produced desireable effect is shown as under Kyron $\mathrm{T}_{134}>$ Kyron $\mathrm{T}_{134}$ + primogel $>$ primogel and for polymers chitosan $>$ chitosan+CMC $>$ chitosan+PVP $>$ CMC $>$ CMC+PVP $>$ PVP (Figure 6).

\section{Discussion}

The finding of our study are substantiated by Diener et al. [7], who reported the disability because of the cognitive decline and recurrent stroke in the patients of ischaemic stroke. The preventive use of telmisartan was not found effective, with no difference between the two antiplatelet regimens [8]. An active, double blind and placebo controlled study has also demonstrate approximately same effects of aspirin with extended release dipyridamole versus clopidogrel and telmisartan on disability and cognitive function after recurrent stroke in patients with ischaemic stroke in the PRoFESS trial:

In addition of that Jaiswal et al. [9] reported the Self microemulsifying drug delivery system (SMEDDS) and solid-SMEDDS of telmisartan with the aimed of to overcome the problems of bioavailability and poor solubility. The findings of this study propose the potential use of solid-SMEDDS and SMEDDS to improve the dissolution. That may eventually enhance the oral bioavailability of poorly water soluble telmisartan through oral route.

Our findings are also supported by Chella et al. [10], who presented a work to improve the solubility of telmisartan using liquisolid compacts. That has poor dissolution profile. To prepare the Liquisolid compacts, the major components used were Avicel PH102 as carrier, Transcutol

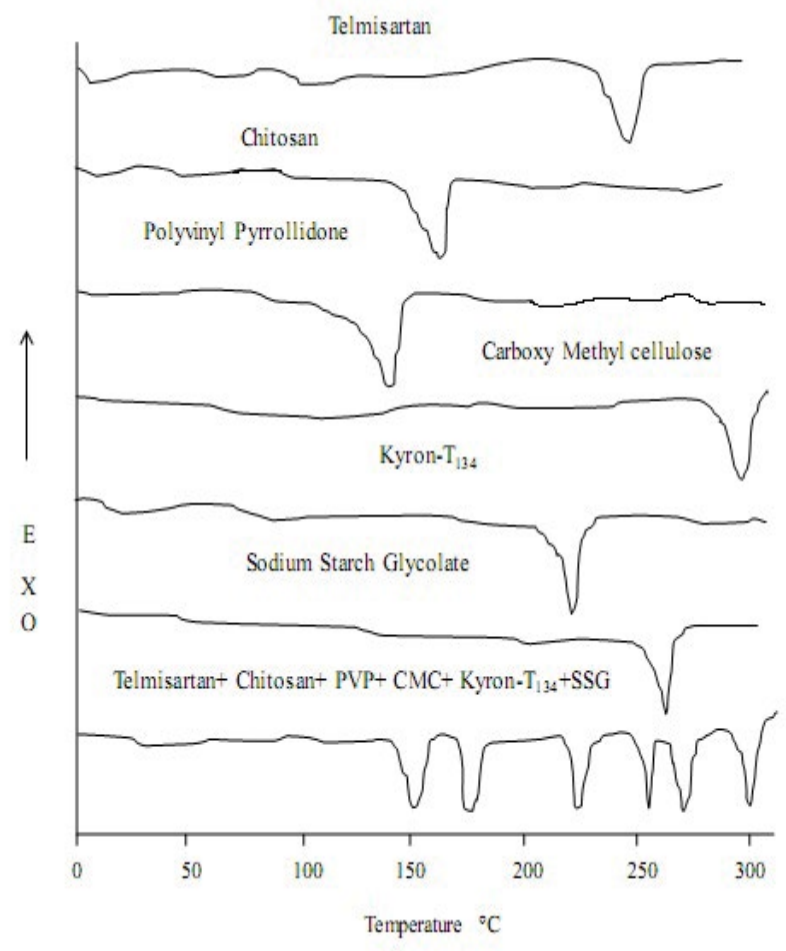

Figure 5: DSC spectra of drug with different excipients.

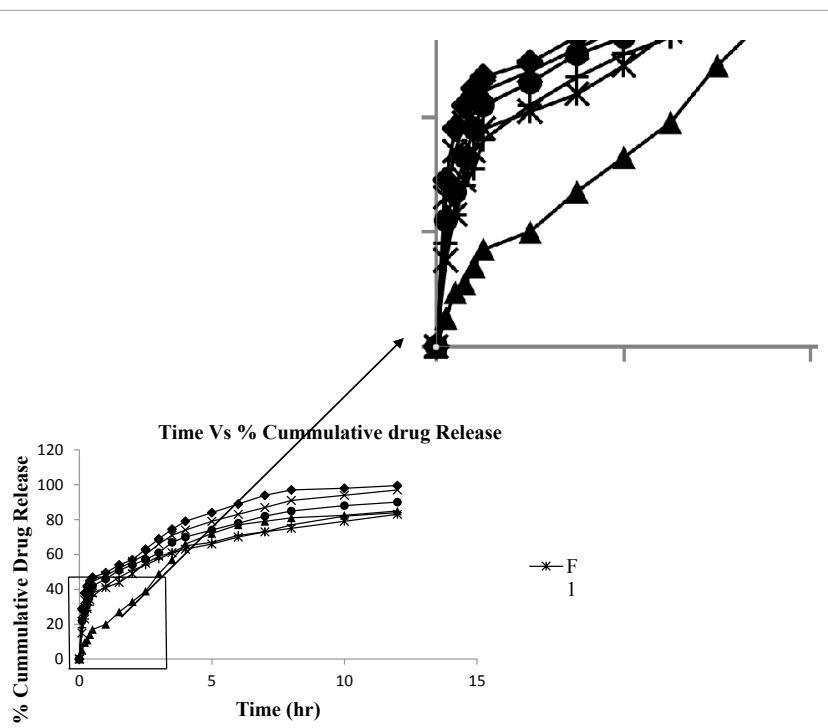

Figure 6: Drug release curves of all 6 formulations upto $12 \mathrm{hrs}$

HP as vehicle, and coating material Aerosil 200. The scientific equipments of DSC, FTIR spectroscopy, various pharmacopoeial tests, angle of repose and X-ray diffraction (XRD) were used to evaluate the formulations. The change in crystallinity of drug, drug excipient interactions, general quality control tests and flow properties of tablets using have also been performed to confirm the study parameters. FTIR studies confirmed, there is no potential interaction between excipients and drug and. The crystallinity of drug was studied by XRD and DSC that showed no change/reduction. After the storage for three months, no significant difference was observed in the drug release profile and tablet properties. Hence, the characterization and preparation of liquisolid compacts may improve the dissolution of telmisartan.

Our findings are also in line with Yang et al. [11] who reported the $\mathrm{pH}$ dependent pattern of Telmisartan release. They tried to improve the $\mathrm{pH}$ dependent release of telmisartan that is a poorly soluble drug through the aminoclay complex formation. Furthermore, drug crystallinity was altered to an amorphous form through the molecular interactions between telmisartan and aminoclay. Telmisartan demonstrated complete dissolution and rapid at $\mathrm{pH} 1.2$ within 15 minutes from all the tested formulations. However, a negligible dissolution at $\mathrm{pH} 4$ and $\mathrm{pH}$ 6.8 was noticed for the untreated powder. While the formation of drug clay complex considerably improved the dissolution rate as well as the extent of drug release at the higher $\mathrm{pH}$ 's. In addition, following an oral administration of telmisartan -aminoclay, $\mathrm{C}_{\max }$ and AUC of telmisartan increased by about 8 and 5 fold respectively, while $\mathrm{T}_{\max }$ was shorten. The results suggest that formation of complex should be promising to enhance the bioavailability of a poorly soluble drug, telmisartan.

\section{Conclusion}

In conclusion, the determinants of MDERTS are adjustable within acceptable range to enhance the efficiency. An extended release profile using response surface methodology may also be designed to formulate the MDT's to render the dose, regimen, protocol and frequency of hypertensive patient in clinical practice.

\section{References}

1. Bhaskar DK, More MR, Sockan GN, Kunchu K, Mani T (2011) Formulation and Evaluation of orodispersible tablets of propranolol hydrochloride. International journal of pharmaceutical research \& development 2: 46-52. 
Citation: Akram MA, Nazir T, Sarfraz M, Nazir SR (2015) Designing, Development and Formulation of Mouth Disintegrating Telmisartan Tablet with Extended Release Profile Using Response Surface Methodology. J Bioequiv Availab 7: 262-266. doi:10.4172/jbb.1000250

2. Kalia A, Shelly K, Bedi N (2009) Formulation and evaluation of Mouth Dissolving tablets of Oxacarbazepine. International Journal of Pharmacy and Pharmaceutical Sciences 1: 12-23.

3. Shojaei AH, Chang RK, Guo X, Burnside BA, Couch RA (2001) Systemic drug delivery via the buccalmucosal route. Pharmaceutical Technology: 70-81.

4. Shah V, Patel S, Jatav RK, Jain A, Sheorey RV (2011) Formulation and evaluation of mouth dissolving tablets of metoclopramide hydrochloride by direct compression technique. Int J Drug Disc Herbal Res 1: 100-103.

5. Kathpalia H, Sule B, Patil A, Mahadik A, Sharma K (2014) Controlled Release Orally Disintegrating Tablets: Int J Pharm Sci Rev Res 24: 35-42.

6. Yang D, Kulkarni R, Behme RJ, Kotiyan PN (2007) Effect of the melt granulation technique on the dissolution characteristics of griseofulvin. Int J Pharm 329: 72-80.

7. Diener HC, Sacco RL, Yusuf S, Cotton D, Ounpuu S, et al. (2008) Effects of aspirin plus extended-release dipyridamole versus clopidogrel and telmisartan on disability and cognitive function after recurrent stroke in patients with ischaemic stroke in the Prevention Regimen for Effectively Avoiding Second
Strokes (PRoFESS) trial: a double-blind, active and placebo-controlled study. Lancet Neurol 7: 875-884.

8. Diener HC, Sacco R, Yusuf S (2007) Rationale, design and baseline data of a randomized, double-blind, controlled trial comparing two antithrombotic regimens (a fixed-dose combination of extended-release dipyridamole plus ASA with clopidogrel) and telmisartan versus placebo in patients with strokes: the Prevention Regimen for Effectively Avoiding Second Strokes Trial (PRoFESS). Cerebrovasc Dis 23: 368-380.

9. Jaiswal P, Aggarwal G, Harikumar SL, Singh K (2014) Development of selfmicroemulsifying drug delivery system and solid-self-microemulsifying drug delivery system of telmisartan. Int J Pharm Investig 4: 195-206.

10. Chella N, Narra N, Rama Rao T (2014) Preparation and characterization of liquisolid compacts for improved dissolution of telmisartan. J Drug Deliv 2014: 692793.

11. Yang $L$, Shao Y, Han HK (2014) Improved pH-dependent drug release and oral exposure of telmisartan, a poorly soluble drug through the formation of drugaminoclay complex. Int J Pharm 471: 258-263. 